\title{
Editorial
}

\section{Topological nanophotonics}

https://doi.org/10.1515/nanoph-2019-0197

Topological phenomena are ubiquitous in physics, with explorations spanning areas from condensed-matter physics to physical cosmology. The 2016 Nobel Prize, awarded to Thouless, Haldane, and Kosterlitz for discoveries embracing topological phenomena [1, 2], has stimulated exciting research leveraging topological concepts in many exciting ways, including topological photonics. Early visionary ideas [3] now manifest in a plethora of novel directions [4-6] that both challenge our curiosity and understanding of light-matter interactions, while also holding profound potential to impact information and communication technologies. While state of the art can roughly be summarized as a broad palette of exciting possibilities, there is natural emphasis on conceptual studies within classical electrodynamics [7], for example, Maxwell's equations accompanied by classical constitutive laws for interactions of light with matter. On the other hand, there is so far less emphasis on exploring topological phenomena in technology-mature nanophotonic platforms, which would allow a fruitful marriage of topological and quantum aspects of light-matter interactions (Figure 1).

Paralleling topological phenomena, spatial and temporal symmetries are likewise inherent to our understanding of complex phenomena and conservation laws in nature. Hermitian-eigenvalue descriptions are well motivated in most solid-state systems of interest, being also at the heart of quantum physics and constituting a foundation for processing of quantum states in emerging quantum technology. On the contrary, non-Hermitian dynamics is often a nuisance associated with detrimental and unavoidable damping due to the environment. As such, non-Hermitian dynamics remained virgin territory until a decade ago [10]. In optics, loss can however be balanced by gain, which opens a doorway to parity-time symmetric photon systems with no counterparts in quantum dynamics of electrons [5, 11]. Remarkably, eigenvalues behind the spectral response and the time dynamics can be entirely real-valued. Parity-time symmetry can support exceptional points, now being more than mathematical subtleties; coalescing eigenstates have intriguing consequences heralded by a breakdown of standard perturbation theory. In this way, the concept of exceptional points opens a new paradigm with unusual light-matter interactions and unprecedented control over quantum emitters and single-photon emission dynamics.

An often emphasized aspect of topological photonics consists in the formation of edge states at topological phase boundaries separating lattice regions of trivial and non-trivial topology. Being topologically protected, such states open a doorway to unidirectional waveguiding of light. Photonic lattices have been realized with the aid of photonic crystal concepts and coupled resonator arrays [12], while two-dimensional materials also offer directions for topological plasmonics [13, 14]; although unidirectionality might sound trivial, in optics it is usually prevented by time-reversal symmetry. Breaking of time-reversal symmetry is therefore of great interest to realize many exciting phenomena in this rapidly developing field. In bridging topological physics and nanophotonics, we anticipate unforeseen topological control of quantum light-matter interactions.

We anticipate developments in topological nanophotonics facilitating intriguing interplays of light, matter, topology, and symmetry (Figure 1F), and it is in this exciting landscape that the idea of this special issue materialized, with several of the contributions originating from an exploratory Workshop on Topological Nanophotonics organized in 2018 by two of us [15]. This special issue contains a collection of exciting papers in this context, providing a snapshot of recent research in the area of topological photonics, and an outlook to its future development. Specifically, Wolff et al. theoretically considered parity-time symmetric systems and explore the dynamics at the exceptional point under the influence of fluctuations caused by inevitable noise [16]. Wang et al. experimentally demonstrated topological protection in spatially entangled biphoton states, using a silicon platform with a lattice of coupled waveguides [17]. Pocock et al. theoretically explored bulk-edge correspondence in topological plasmonic chains in the context of long-range hopping [18]. Kudyshev et al. theoretically explored the use of dynamically tunable resonators to enable photonic topological phase transitions on demand [19]. Xia et al. theoretically showed that both transverse electric and transverse magnetic designer surface plasmons can be supported at the interface between a suitably designed bianisotropic metamaterial. [20]. Christiansen et al. considered the design of photonic topological insulators as an inverse design problem, 


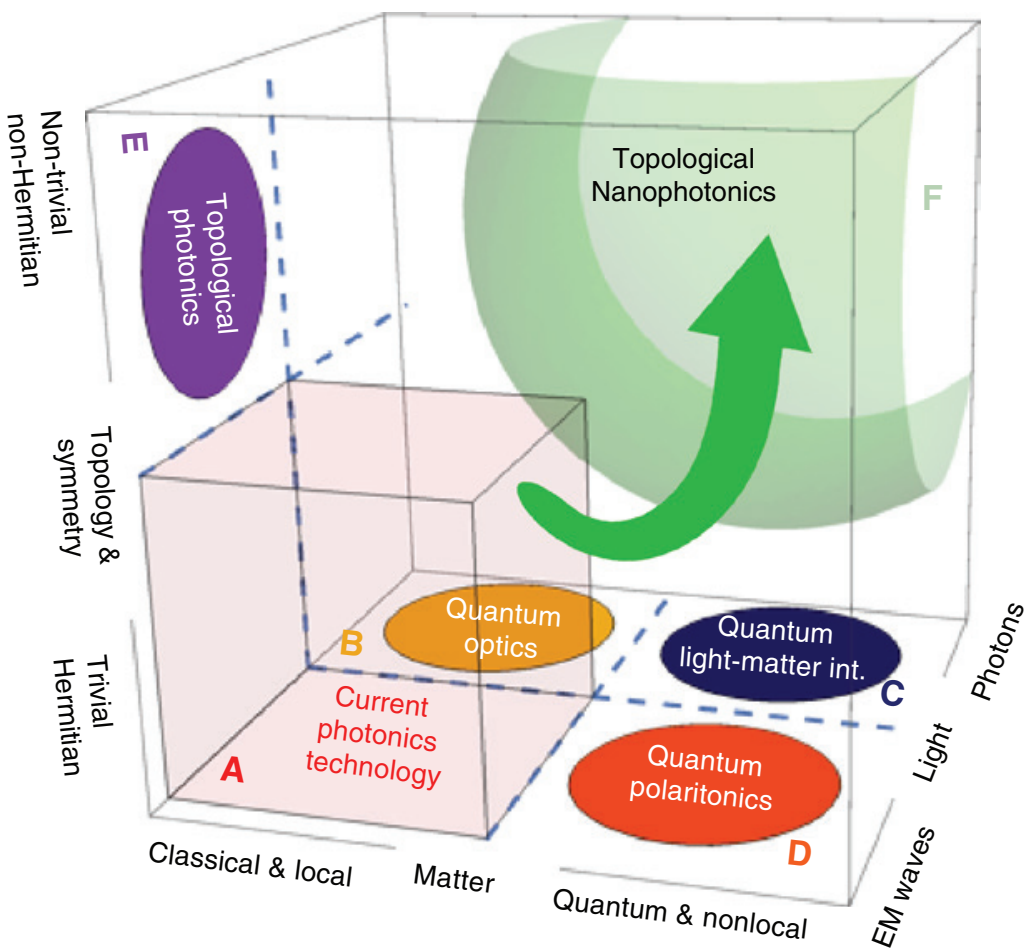

Figure 1: Rooted in classical electrodynamics, current photonics (A) constitutes key enabling technology (fiber-optics, lasers, detectors, integrated photonics, etc.), being critical to Information and Communications technology. Emerging quantum technologies harvest from quantum phenomena of both light (B) and matter (D), and combined light-matter aspects [8, 9] (C), while there is growing attention to classical electrodynamics in systems comprising non-trivial topologies and symmetries [7] (E). Developments in topological nanophotonics will enable explorations of the virgin territory (F) hosting intriguing interplays of light, matter, topology, and symmetry.

using topology optimization tools [21]. Hayran et al. theoretically explored the coupling of plane waves into topological one-way surface waves with prospects for topologically protected wave-rerouting around an electrically large impenetrable object of complex shape [22]. Zdagkas et al. explored exact propagating solutions of Maxwell equations in the form of single-cycle (termed flying doughnuts by the authors), space-time non-separable toroidal pulses and showed that they exhibit a complex and robust fine topological structure [23]. Lannebére and Silveirinha explored photonic analogues of the Haldane and Kane-Mele models for electrons in condensed-matter systems [24]. Van Mechelen and Jacob developed a unified perspective of unidirectional topological edge waves in non-reciprocal media, while focusing on the role of photonic spin in non-reciprocal gyroelectric media [25]. Fan et al. theoretically explored topological interface states in two-dimensional boron-nitride-graphene heterostructure [26]. Finally, Orazbayev and Fleury theoretically and numerically studied the robustness to disorder of subwavelength edge modes in topological systems [27]. This special issue provides a vista on recent efforts in this exciting and rapidly growing area of research, with the hope of inspiring new concepts and experimental efforts to translate these ideas into nanophotonic implementations with exotic optical transport for new technological endeavors.

Acknowledgments: NAM is a VILLUM Investigator supported by VILLUM FONDEN (grant no. 16498). SIB acknowledges the European Research Council (grant 341054). AA has been supported by the Air Force Office of Scientific Research and the Simons Foundation.

\section{References}

[1] Haldane FDM. Nobel lecture: topological quantum matter. Rev Mod Phys 2017;89:040502.

[2] Kosterlitz JM. Nobel lecture: topological defects and phase transitions. Rev Mod Phys 2017;89:040501. 
[3] Lu L, Joannopoulos JD, Soljačić M. Topological photonics. Nat Photon 2014;8:821-9.

[4] Khanikaev AB, Shvets G. Two-dimensional topological photonics. Nat Photon 2017;11:763-73.

[5] El-Ganainy R, Makris KG, Khajavikhan M, Musslimani ZH, Rotter S, Christodoulides DN. Non-Hermitian physics and PT symmetry. Nat Phys 2018;14:11-9.

[6] Ozawa T, Price HM, Amo A, et al. Topological photonics. Rev Mod Phys 2019;91:015006.

[7] Mittal S, DeGottardi W, Hafezi M. Topological photonic systems. Opt Photon News 2018;29:36-43.

[8] Bozhevolnyi SI, Mortensen NA. Plasmonics for emerging quantum technologies. Nanophotonics 2017;6:1185.

[9] Fernández-Domínguez AI, Bozhevolnyi SI, Mortensen NA. Plasmon-enhanced generation of nonclassical light. ACS Photonics 2018;5:3447-51.

[10] Bender CM. Making sense of non-Hermitian Hamiltonians. Rep Prog Phys 2007;70:947.

[11] Özdemir SK, Rotter S, Nori F, Yang L. Parity-time symmetry and exceptional points in photonics. Nat Mater 2019;18. doi: 10.1038/ s41563-019-0304-9.

[12] Hafezi M, Mittal S, Fan J, Migdall A, Taylor JM. Imaging topological edge states in silicon photonics. Nat Photon 2013;7:1001-5.

[13] Jiang B-Y, Ni G-X, Addison Z, et al. Plasmon reflections by topological electronic boundaries in bilayer graphene. Nano Lett 2017;17:7080-5.

[14] Hasdeo EH, Song JCW. Long-lived domain wall plasmons in gapped bilayer graphene. Nano Lett 2017;17:7252-7.

[15] Workshop on Topological Nanophotonics, Odense, Denmark, 2018. (Accessed July 1, 2019, Available at http://mortensen-lab.org/topologicalnanophotonics.pdf).

[16] Wolff C, Tserkezis C, Mortensen NA. On the time evolution at a fluctuating exceptional point. Nanophotonics 2019;8:1319-26.

[17] Wang M, Doyle C, Bell B, et al. Topologically protected entangled photonic states. Nanophotonics 2019;8:1327-35.

[18] Pocock SR, Huidobro PA, Giannini V. Bulk-edge correspondence and long-range hopping in the topological plasmonic chain. Nanophotonics 2019;8:1337-47.

[19] Kudyshev ZA, Kildishev AV, Boltasseva A, Shalaev VM. Photonic topological phase transition on demand. Nanophotonics 2019;8:1349-56.

[20] Xia L, Yang B, Guo Q, et al. Simultaneous TE and TM designer surface plasmon supported by bianisotropic metamaterials with positive permittivity and permeability. Nanophotonics 2019;8:1357-62.

[21] Christiansen RE, Wang F, Sigmund O, Stobbe S. Designing photonic topological insulators with quantum-spin-hall edge states using topology optimization. Nanophotonics 2019;8:1363-9.

[22] Hayran Z, Gangaraj SAH, Monticone F. Topologically protected broadband rerouting of propagating waves around complex objects. Nanophotonics 2019;8:1371-8.

[23] Zdagkas A, Papasimakis N, Savinov V, Dennis MR, Zheludev NI. Singularities in the flying electromagnetic doughnuts. Nanophotonics 2019;8:1379-85.

[24] Lannebére S, Silveirinha MG. Photonic analogues of the Haldane and Kane-Mele models. Nanophotonics 2019;8:1387-97.

[25] Van Mechelen T, Jacob Z. Unidirectional Maxwellian spin waves. Nanophotonics 2019;8:1399-416.

[26] Fan Z, Dutta-Gupta S, Gladstone R, et al. Electrically defined topological interface states of graphene surface plasmons based on a gate-tunable quantum Bragg grating. Nanophotonics 2019;8:1417-31.

[27] Orazbayev B, Fleury R. Quantitative robustness analysis of topological edge modes in C6 and Valley-Hall metamaterial waveguides. Nanophotonics 2019;8:1433-41.

\section{N. Asger Mortensen}

Center for Nano Optics, University of Southern Denmark, Campusvej 55, DK-5230 Odense M, Denmark; and Danish Institute for Advanced Study, University of Southern Denmark, Campusvej 55, DK-5230 Odense M, Denmark, e-mail: asger@mailaps.org. https://orcid.org/0000-0001-7936-6264

\section{Sergey I. Bozhevolnyi}

Center for Nano Optics, University of Southern Denmark, Campusvej 55, DK-5230 Odense M, Denmark; and Danish Institute for Advanced Study, University of Southern Denmark, Campusvej 55, DK-5230 Odense M, Denmark, e-mail: seib@mci.sdu.dk

\section{Andrea Alù}

Photonics Initiative, Advanced Science Research Center, City University of New York, New York, NY 10031, USA; Physics Program, Graduate Center, City University of New York, New York, NY 10026, USA; and Department of Electrical Engineering, City College of New York, New York, NY 10031, USA, e-mail: aalu@gc.cuny.edu 